\title{
Research Progress and Application in Aeronautics of Flow Control Technology for Increasing Lift and Reducing Drag
}

\author{
Limin Song, Yuanyuan Sun, Yongxue Liu, Lei Ming, Shuqiang Wang and Duo Wang \\ Aviation University of Air Force, \\ Changchun, Jilin, China \\ liminsong_2001@163.com
}

\begin{abstract}
Flow control technique is an important research field of aerodynamics. Flow control technique uses the interactions of fluid hydrodynamic to control and amplify the flow signal by changing the local flow. As far as possible to optimize the flow field distribution, increase the lift, reduce the drag and effectively improve the lift drag ratio, are also the important research subjects in aircraft design. As the requirements of plane design performance improving constantly, the fluid flow control becomes more and more important, and shows the irreplaceable role. In this paper the passive flow control method and active flow control method with applications to increasing lift and reducing drag are introduced in detail. The conclusion is active flow control's effect is more obvious compared by the passive flow control means. The working keys and directions are put forward in the paper.
\end{abstract}

Keywords- passive flow control; active flow control; drag reduction; high lift

\section{INTRODUCTION}

The boundary layer theory which Prandtl proposed in 1904 has greatly promoted the development of fluid mechanics and aerodynamics. Boundary layer is called the attachment surface layer. Due to air viscosity and not smooth surface, a layer fluid of the object surface with can be affected by surface drag, thus flow velocity is reduced to zero. While one layer is involved by the other layer, the velocity of fluid layer will increase along the surface normal. The thin layer is called the boundary layer. The boundary layer is divided into the boundary layer of laminar flow and boundary layer of turbulent flow. The friction resistance of turbulent boundary layer is greater. Due to the adverse pressure gradient, the back and forward air will meet and arch from the wing surface, thereby generating vortex area, as shown in figure 1 and 2 . The bigger vortex area will not only have a greater pressure difference resistance, but also the less pressure difference of upper and lower wing surface will reduce the lift. So the flow control technology in aviation reaches a high lift and drag reduction, according to the boundary layer and vortex control, improves flight performance.

Flow control can be divided into passive flow control and active flow control. Passive flow control technology starts earlier, already has widely engineering application.
Active air flow control has a late start, but also become a more systematic research field.

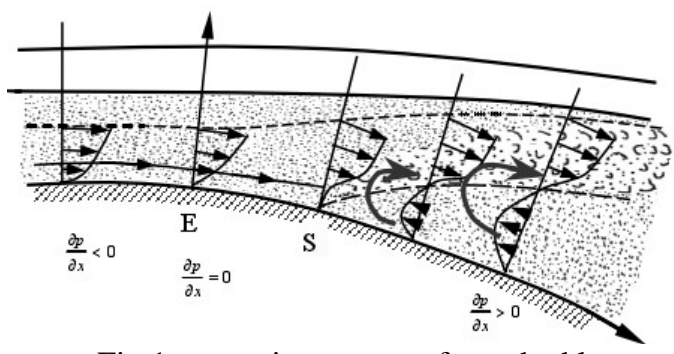

Fig.1 separation reason of attached layer

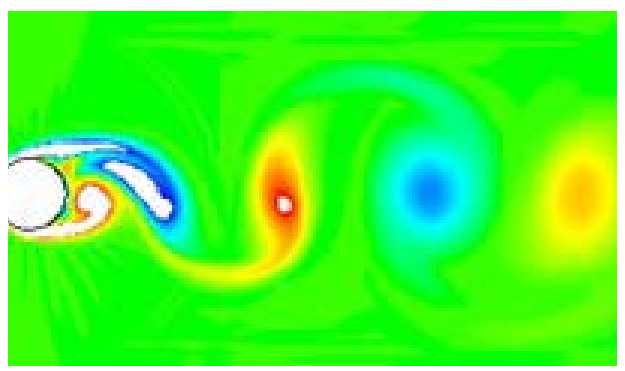

Fig.2 separation process of attached layer

\section{MECHANISM AND APPLICATION OF PASSIVE FLOW CONTROL TECHNOLOGY}

Passive control is a control technology which changes the flow environment through the passive flow control device. Passive control is also called fixed control, and its effect is easily affected by the changes of the fluid movement state. Because the wing is the main components to generate lift for aircraft, the flow control mainly used to change the flow of wing boundary layer and delay the flow separation of wing boundary layer, so as to achieve the purpose of increasing lift and drag reduction.

Wing fence is the most simple passive flow control device, it can change the boundary layer flow and delayed flow separation on tip to reduce the resistance and increase lift, through the boundary layer barrier from wing root to wing tip pushed. The leading edge flap and trailing edge flap are also widely used on plane to increase lift, but the result of resistance is inevitable. $\mathrm{Choi}^{[1]}$ made numerical simulation on groove surface turbulent fluid flow with DNS 
model in 1993, and showed that grooves can reduce resistance. Professor Reif received inspiration by observing the shark swimming, it is proved by test that the grooves on the shark skin can make the boundary layer stability, which can change the wall surface to achieve good effect of drag reduction. A lot of researches and experiments ${ }^{[2]}$ showed, small grooves aligned with the flow direction can effectively reduce friction resistance of turbulent boundary layer, and it has been applied to large aircrafts.

In addition, from the plano-convex airfoil to the supercritical airfoil, the design and research of airfoil has been basic research topic in the field of aircraft design for a long time. The supercritical airfoil is beneficial to reducing the separation of boundary layer degree and delaying the occurrence of the shock wave, so as to reducing aircraft weight, improving flight performance. Li Weijie ${ }^{[3]}$ made the improved design of humpback whale non smooth leading edge protrusions to the NACA63-210 wing based on the basic principles of bionics on the basis of the study of the foreign scholars. Through the numerical simulation, the conclusion is that the bionic wing can make the lift coefficient increase and make the resistance decrease apparently in addition to $0^{\circ}$ angle of attack. Yang Yang ${ }^{[4]}$ also proved by experiment, the bumps design of supercritical airfoil can reduce the shock resistance of supersonic flight and improve the lift-drag ratio. In addition, the position, shape and distribution of bumps will affect the drag reduction effect.

In 1947, Taylor $^{[5]}$ proposed the concept of vortex generator, and proved that it can be used in boundary layer flow control. In fact the basic working principle of vortex generator is following: It is arranged in a mounting angle on the body surface, and produces strong wingtip vortex due to its small the lift-drag ratio. It can effectively control the flow separation as the energy delivered to the low energy boundary layer. The test results show that, the vortex generator used in multi element airfoils, can increase the stall angle of attack under the take-off, landing states, and achieve greater maximum lift coefficient.

Due to tip vortices of the 3-dimensional wing (as shown in Fig.3), an aircraft generates lift and also produces resistance in flight. The resistance is known to induce resistance. While the drag reduction control of the induced drag is also an important research work, especially has the research value for large aircraft. At present the main methods is the tip winglet or the wing tip sails. Liu Xiaolei ${ }^{[6]}$ designed a wingtip winglets based RAE2822 supercritical airfoil. The numerical simulation results show that the wingtip vortex strength is effectively reduced to increase lift and drag reduction after equipping with the winglet (as shown in Fig.4).

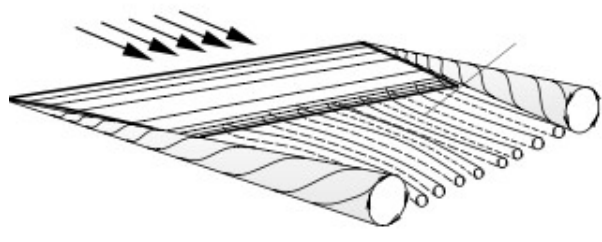

Fig.3 generation of wing tip vortex

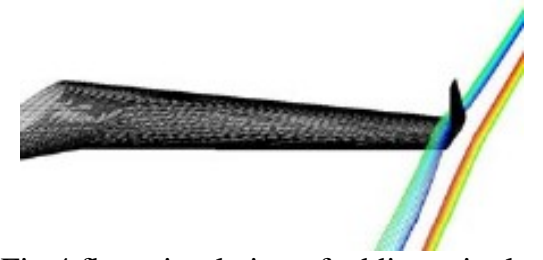

Fig.4 flow simulation of adding winglets

\section{MECHANISM AND APPLICATION OF ACTIVE FLOW CONTROL TECHNOLOGY}

Active flow control is also called dynamic control. It is coupled applying appropriate perturbation model and flow internal patterns to achieve flow control in flow field. Active flow control technology belongs to the emerging field. It will have a major impact on design concepts and methods of aerodynamic layout in the future. The active flow control technology closely combines with modern information technology, materials technology, electromagnetic technology, MEMS technology, promoting rapid development of the active flow control technology to the direction of more easy to implement project.

The active flow control mainly attracts flow of the upper wing surface and blows the vortex of wing trailing edge region to achieve early. Now the development of the synthetic jet technology has more obvious effects. This paper briefly introduces the two kinds of active control technology combining methods with electromagnetic technology and MEMS technology.

\section{A. The electromagnetic Force of Flow Control}

The electromagnetic force is the force's general term of a charge or current in the electromagnetic field. The force of a current-carrying conductor in a magnetic field is also called electromagnetic force. The electromagnetic force is a kind of volume force, which is expressed the magnetic induction intensity B and current density $\mathrm{J}$.

The following equation:

$$
f=J \times B
$$

Therefore, the incompressible fluid control equation can be written as:

$$
\frac{\partial \mathrm{U}}{\partial t}+U \cdot \nabla U=-\nabla P+\frac{1}{R e} \nabla^{2} U+S_{t}(J \times B)
$$

In the formula, $\mathrm{U}$ is the speed of a fluid motor, $\mathrm{P}$ is the pressure of flow field, Re is the Reynolds number, $S_{t}$ as 
function coefficient equal to the ratio of the electromagnetic force strength and fluid flow inertia.

The electrode strip and magnetism strip are alternatively arranged to form an activation plate, placed the flow of the electrolyte solution, as shown in figure 5 . Lorenz force has the same direction with the fluid flow. T he Lorenz force can change the boundary layer structure, thus to control the fluid.

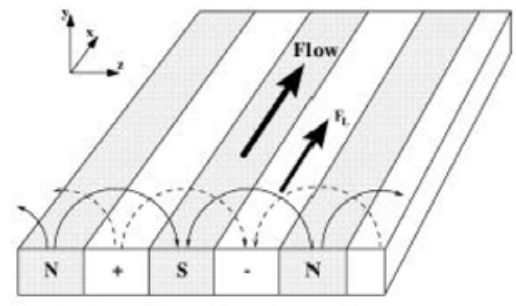

Fig.5 flowing medium electromagnetic activation plate

Early in the middle of the twentieth Century, people try to use electromagnetic force characteristic to control low conductive fluid flow, and has carried the research. The experiment proved that the electromagnetic force can effectively eliminate vortex and reduce drag.

$\operatorname{Reed}^{[7]}$ found magnetic in addition can suppress turbulence, even can make it laminar flow again and significantly reducing the flow resistance. Gardner ${ }^{[8]}$ analysised and validated that the electromagnetic force can obviously reduce the flow resistance through pipe flow mechanism of the magnetic generator. Gailitis ${ }^{[9]}$ composed the electromagnetic field activation plate which strip electrode and magnetic poles are alternatively arranged. It is immersed in the flow of weak electrolyte solution, and the resulting Lorentz force can change the boundary layer structure. Subsequently, Berger ${ }^{[10]}$ studied the boundary layer in the electromagnetic field by numerical simulation and related experiments. The electromagnetic force to drag reduction in turbulent boundary layer research is paid attention by the researchers ${ }^{[11,12,13]}$ in recent years. Zhang $\mathrm{Hui}^{[14]}$ inserted the column on the surface coating electromagnetic activation plate into the flow of electrolyte solution. When the electromagnetic plate has not been activated, the fluid in the cylinder surface somewhere formed vortices. After the electromagnetic plate has been activated, Lorentz force makes the boundary layer structure produces change apparently near the flow field of the cylindrical surface, and fluid separation point on the wall disappeared with vortex suppression. Liu Huixing ${ }^{[15]}$ puts the rudder coated electromagnetic activation plate on surface into the solution. The fluid boundary layer structure is controlled by the electromagnetic force on the rudder surface. It was found that a certain intensity and intermittent frequency spanwise electromagnetic force can effectively reduce the rudder's fluid resistance of the turbulent state. Based on the electromagnetic field and the basic equations of fluid mechanics, Mei Dongjie ${ }^{[16]}$, studied the spanwise oscillating electromagnetic force of the turbulent channel flow experimentally and numerically. It shows interaction the streamwise vortex of the electric magnetic induced and wall turbulence. Shi Weiping ${ }^{[17]}$ simulated the process of the flow around a circular cylinder in electromagnetic field using a lattice Boltzmann method, and studied cylindrical resistance force is impacted by electromagnetic force. The results showed that the electromagnetic force can change the boundary layer structure of flow around cylinder, delaying boundary layer separation, while suppression of vortex shedding and reducing drag.

To sum up, the electromagnetic force generated through electromagnetic field in a conductive fluid boundary layer has distinct distribution features, and can effectively change the boundary layer structure, control flow separation of boundary layer. Though changing arrangement of the electrode and a magnetic stripe, different directions electromagnetic forces are generated. Therefore, the electromagnetic force control is easy to realize, and its flexibility is also very strong. It is important for military application to develop the turbulent boundary layer drag reduction technology of electromagnetic force.

\section{B. The Plasma of Flow Control}

Plasma is also called electric slurry. It is ionized gas substance which composed by a part of electronics after deprivation of atom and positive and negative electrons of atom ionized. Plasma is a good conductor. Roth ${ }^{[18,19]}$ early worked for the study of plasma to assist drag reduction. Yano ${ }^{[20]}$ also use wind tunnel test to ultrasound unequilibrium plasma, Shang conducted on supersonic aircraft plasma drag reduction experiment. Luo Jinling ${ }^{\text {[21] }}$ made plasma numerical simulation on high speed aircraft. It is indicated that the plasma can significantly reduce the flight resistance under certain conditions. The drag reduction effect is also not same to different Mach numbers.

It is showed on research, plasma flow control technique can make use of static electricity to control the boundary layer flow, and effectively improve the flow separation condition, thereby reducing the coefficient of drag in flight. The test results show, plasma technology can make the flight resistance reduce about 30\%. But these experiments are under the glow discharge conditions. To form plasma on the surface of the aircraft still need be researched and experimented to experts. Plasma hydrodynamics can be not only used for flow control to reduce drag, also be used in stealth technology.

\section{CONCLUSIONS AND PROSPECTS}

Through the analyses, active flow control and passive flow control can control fluid flow to some extent, but the active flow control has more obvious advantage. Passive control is predetermined. when flow conditions deviate from the design state, it is impossible to achieve the best control effect. Active control technology can change the flow 
structure of the full flow field. Active flow control has the advantage of appearing in the need time and site, and changes the local or global flow effectively through the local energy input. At the same time, the aircraft flight performance was improved. But the research is just a beginning, and active flow control technology is facing difficulties from the theory to the practice. To achieve the purpose of practical application, there are a lot of efforts and works to do.

In addition, the object of the active flow control is boundary flow and other related phenomena, which is currently the aerodynamics research problem. It is not easy to establish the exact models. So the exact model of active flow control technology is one problems of solved urgently.

\section{REFERENCES}

[1] Choi K, Orchard D. Turbulence management using riblets for heat and mom-entumtransfer [J]. Thermal Fluid Sci., 1997, 15: 109- 124.

[2] Bechert D W, Bruse M, Hage W. Fluid mechanics of biological surfaces and their technological application. [J] NaturwissenSchaften,2000,87(4):157-171

[3] Li Weijie. Numerical Simulation of Bionic Wing for Drag Reduction, [D]Changchun: Jilin Univercity,2008

[4] Yang Yang,Chen Yingchun,Huang Wei. Active Drag-reducing Technique Using Bumps on Transonic Wings, [J]Civil Aircraft Design and Research ,2012,02:13-18

[5] Taylor H D. Elimination of Diffuser Separation by Vortex Generators,United Airfcraft Corporation Report, NoR-4012-3,1947

[6] Li Xiaolei. Numerical Simulatian of 11Vingfet Characteristics far Drag Reduction, [D]Changchun: Jilin Univercity,2010

[7] Reed C.B. and Lykoudis P.S. The effect of a transverse magnetic field on shear turbulence. J Fluid Mech. Vol.89:p.147.

[8] Gardner R.A. and Lykoudis P.S. Magneto-fluid-mechanic pipe flow in a transverse magnetic field. Part I. Isothermal flow. J Fluid Mech. Vol.47.p.737.

[9] Gailitisa A, Lielausis O. On a possibility to reduce the hydrodynamical resistance of a plate in an electrolyte [J] . Applied Magnetohydrodynamics, 1961,12:143-146.

[10] BERGER T, KIM J, LEE C , et al. Turbulent boundary layer control utilizing the Lorentz force [J] . Physics of Fluids,2000, 12 (3):631649.
[11] Crawford C.H. and Karniadakis G.E. Reynolds stress analysis of EMHD-controlled wall turbulence. Part I. Streamwise forcing. Phys. Fluids,1997, 9 (3), 788:806

[12] R.Vilela mendes \& J.A. Dente, Boundary-Layer Control by electric fields, Journal of Fluids Engineering, Vol.120, 1998, P.626

[13] Yiqing Du, V.Symeonidis,\& G.E.Karniadkis, Drag reduction in wallbounded turbulence via a transverse traveling wave, J Fluid Mech. 2002,Vol.457.p.1-34

[14] ZHANG Hui, FAN Baochun, CHEN Zhi-hua, Cylinder Wake Flow Affected by Width of Electro-magentic Actuator, Engineering Mechanics, Vol.24(12), 2007, P.164-167

[15] LIU Hui-xing,ZHOU Ben-mou,LIU Zong-kai,LUOYibing,CHENGFeng,Drag Reduction of Rudder Controlled by Spanwise Electromagnetic Force, Journal of Hebei University (Natural Science Edition) , Vol.30(5), 2010, P.485-489

[16] Mei Dongjie ,Fan Baochun, et al. Mechaniam of Drag Reduction by Spanwise Oscillating Lorentz Force in Turbulent Channel Flow, Chinese journal of theoretical and applied mechanics,Vol.43(4), 2011, P.653-659

[17] Shi Wei-ping, Li Xiu-wen, He Peng, Lattice Boltzmann Simulation of Drag Reduction for the Flow around Circular Cylinder in Electromagnetic Field, Journal of Jilin University:Sci Ed, Vol.49(4), 2011, P.575-579

[18] J.R.Roth. Interaction of Electromagnetic Fields with Magnetized Plasmas[R] .AD2A85496. 1996: 204 209

[19] J.R.Roth. Interaction of a uniform glow dicharge plasmain atmosphericair[R] .AD2A296928. 1995

[20] R.Yano,V. contini , etal. Supersonic nonequilibrium plasma wind tunnel measurements of shock modification and flow visualization.AIAA Journal 2000,38 (10): 1879 1887

[21] Luo Jinling, Xu Min, Dai Wuye, Liu Zhen. Numerical Simulatian Investigation on Plama Injection for Drag Reduction of Hypersonic Vehicle, Journal of Astronautics. Vol.30(1), 2009, P.119-122

[22] Henoch, C and Stace, J. Experimental investigation of a salt water turbulent boundary layer modified by an applied streamwise magnetohydrodynamic body force. Phys. Fluids 7,1371-1383, 1995

[23] Crawford, C.H. and Kamiadakis,G.E. Rynolds stress analysis of EMHD controlled wall turbulence, Part I streamwise forcing. Phys. Fluids 9, 788-806, 1997

[24] Weier, T., Gerbeth, G., Mutschke, G., Platacis, E. and Lielausis, O. Experiments on cylinder wake stabilization in an electrolyte solution by means of electromagneticforces localized on the cylinder surface. Experimental Thermal and Fluid Science16,84-91, 1998

[25] Posdziech,O and Grundmann, R. Electromagnetic control of seawater flow around circular cylinders. Eur. J.Mech.B.Fluids 20, 255-274, 2001 